Pathologe 2022 - 43:57-62

https://doi.org/10.1007/s00292-021-00986-x

Angenommen: 24. August 2021

Online publiziert: 5. Oktober 2021

○ Der/die Autor(en) 2021, korrigierte Publikation 2021

Schwerpunktherausgeber

H.I. Grabsch, Maastricht, Niederlande

R. Langer, Linz, Österreich

M. Vieth, Bayreuth

\section{In diesem Beitrag}

- Was sind synoptische Berichte?

- Standardisiert, strukturiert, synoptisch?

- Vollständigere Berichte durch synoptisches Format

- Quellen für synoptische Tumorprotokolle

- Informationstechnische Umsetzung

- ICCR-Protokolle für gastrointestinale Tumoren

- Ausblick

\title{
Standardisierte strukturierte Befundberichte gastrointestinaler Tumoren
}

\author{
Ekkehard Hewer ${ }^{1}$ (D) - Anna Rump ${ }^{2} \cdot$ Rupert Langer ${ }^{2}$ (D) \\ 'Institut universitaire de pathologie, Centre hospitalier universitaire vaudois (CHUV) et Université de \\ Lausanne, Lausanne, Schweiz \\ ${ }^{2}$ Institut für Pathologie und Molekularpathologie, Kepler Universitätsklinikum und Johannes-Kepler- \\ Universität, Linz, Österreich
}

\section{Zusammenfassung}

Synoptische oder strukturierte Berichte in der Medizin, speziell in der Pathologie, sind im Gegensatz zu traditionellen narrativen Berichten gekennzeichnet durch ein listenbzw. laborwertartiges Format und die Verwendung standardisierter Checklisten. Sie tragen zur Vollständigkeit und Verständlichkeit der Berichte und damit letztlich zu einer verbesserten Patientenversorgung bei. Für die Verwendung in der Pathologie publizieren aktuell 2 größere Institutionen Vorlagen für synoptische Berichte, das College of American Pathologists (CAP) und die International Collaboration for Cancer Reporting (ICCR). Synoptische Berichtsvorlagen sind für alle häufigeren Tumortypen verfügbar. Sie bieten nicht nur eine standardisierte Nomenklatur und Checklisten für vollständige Berichte, sondern unterstützen auch eine einheitliche Anwendung diagnostischer Kriterien. Außerdem beinhalten die Protokolle sowohl von CAP als auch von ICCR umfangreiche und aktuelle Referenzen und sind damit eine wertvolle Quelle von Zusatzinformationen, selbst wenn kein synoptisches Berichtsformat verwendet wird. Nutzen und Herausforderungen bei der Implementation von synoptischen Berichten werden diskutiert, insbesondere im Hinblick auf die deutschsprachige Pathologie.

\section{Schlüsselwörter}

Checkliste · Datengenauigkeit · Qualitätssicherungsmaßnahmen im Gesundheitswesen . Pathologie · Qualitätskontrolle

Wer kennt sie nicht: die Anrufe eines klinischen Kollegen, ob man sich nicht bitte noch zu diesem Parameter Stellung nehmen könne, der im Bericht leider fehle, aber doch ungemein wichtig sei. Merkwürdig nur, dass sich keiner seiner Fachkollegen je dafür interessiert und es dazu nur eine einzige Studie von zweifelhafter Qualität gibt - so ist „personalisierte Medizin" eigentlich nicht gedacht! Nicht nur für derlei Probleme, sondern allgemein für die Frage, was in einen Bericht gehört und wie man es formulieren sollte, bietet das Konzept der synoptischen Berichte interessante Lösungsansätze

Eine Standardisierung von Kommunikation kann einen wichtigen Beitrag zur Si- cherheit in der Medizin leisten. Wesentliche Aspekte einer solchen standardisierten Kommunikation sind einerseits die Vollständigkeit der kommunizierten Parameter (z. B. im Rahmen einer Checkliste, die vor Beginn einer Operation im Rahmen eines "team timeout" durchgegangen wird), andererseits eine eindeutige und unmissverständliche Terminologie. In der Pathologie bietet sich hierfür das Konzept der "synoptischen“ (oder auch „strukturierten" bzw. "standardisierten“) Berichte an. Anders als z. B. in den Vereinigten Staaten oder den Niederlanden scheinen sie im deutschen Sprachraum bislang wenig verbreitet zu sein, auch wenn systematische Erhebungen hierzu fehlen. Dieser Übersichtsartikel diskutiert das Konzept der 


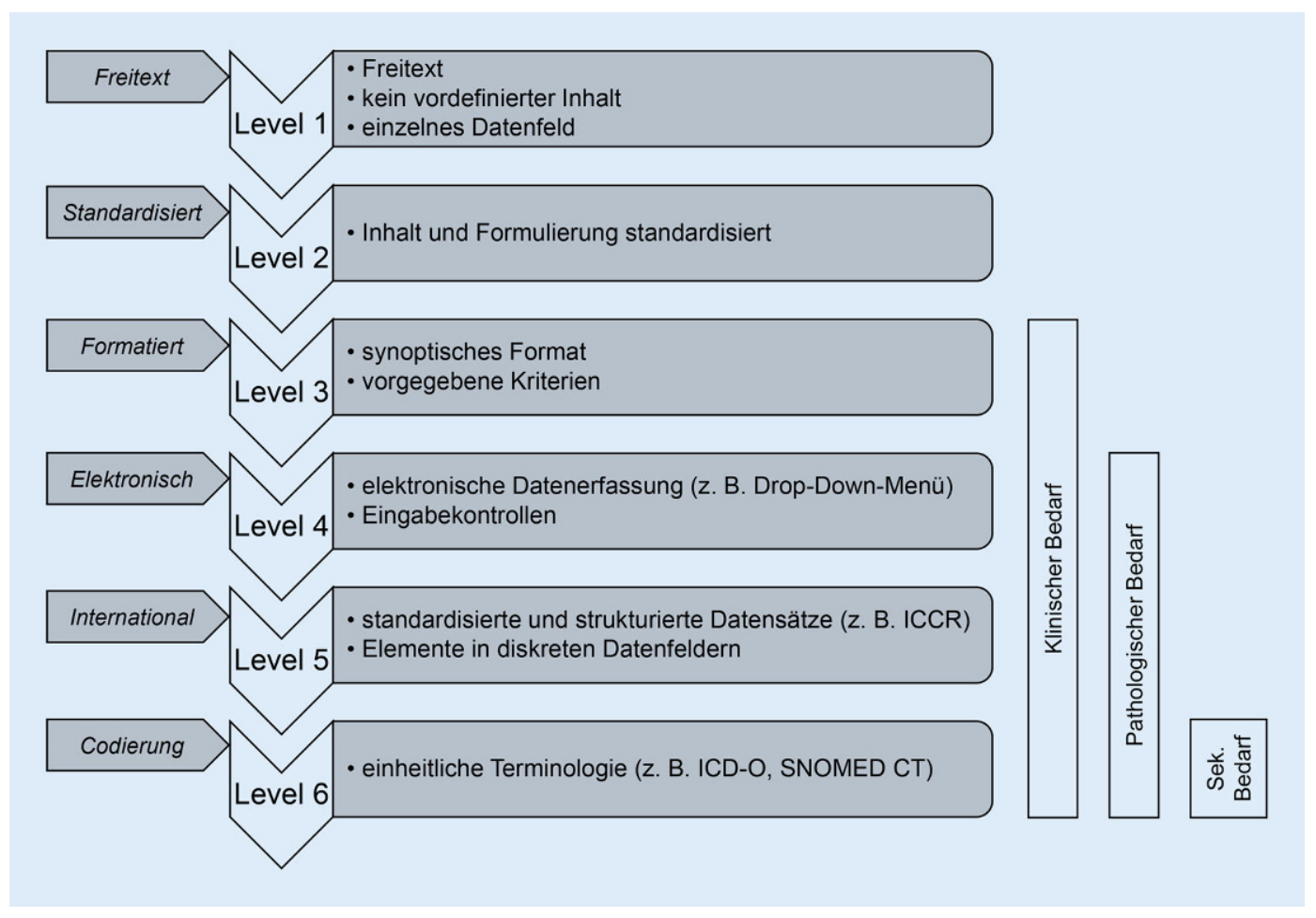

Abb. $1<$ Schematische Darstellung einer stufenweise zunehmenden Standardisierung von Pathologiebefunden. (Modifiziert nach Ellis und Srigley [4]) standardisierten Berichte, die Datenlage zu deren Nutzen und Limitationen sowie Aspekte der praktischen Umsetzung.

\section{Was sind synoptische Berichte?}

Einem Bonmot zufolge, das ursprünglich auf eine andere Berufsgruppe gemünzt war, sich aber durchaus auf die unsrige übertragen lässt, müsse man sich eine Pathologin oder einen Pathologen vorstellen als eine „Person, die eher die Zahnbürste ihres Kollegen als dessen Terminologie benutzen würde". In der Tat kennen wir wohl alle Beispiele terminologischer Eigenheiten, die die Verfasserin oder den Verfasser eines Pathologieberichts schnell preisgeben und die nicht selten mit einiger Vehemenz verteidigt werden. Man müsse eben genau so und so sagen, weil man damit etwas Bestimmtes gleich mit einschließe oder weil es eine elegante Möglichkeit sei, sich nicht auf dieses oder jenes festlegen zu müssen. Bei aller Sympathie für eine elaborierte und differenzierte Sprache ist eine uneinheitliche medizinische Terminologie jedoch eine potenzielle Quelle von Missverständnissen und womöglich daraus resultierenden fehlerhaften Entscheidungen.
An diesem Punkt setzt das Konzept der synoptischen Berichte an: Die jeweiligen organ- und /oder tumortypspezifischen Protokolle definieren eine eindeutige Terminologie, nicht nur für jeden der betreffenden diagnostischen oder prognostischen Parameter (z. B. „Dysplasia“), sondern auch für dessen Status („not identified“ oder "present ${ }^{\prime \prime}$ ) sowie für etwaige nähere Spezifizierungen ("grade“" „lowgrade" oder "high-grade"; "type": "squamous" oder "columnar/barrett").

Die präziseste Definition synoptischer Berichte stammt vom College of American Pathologists (CAP) und umfasst folgende Aspekte [3]:

- Ein listen- bzw. laborwertähnliches Format für jeden Parameter, bestehend aus der Bezeichnung des Datenelements („required data element ${ }^{\prime \prime}$ ) und der Antwort („,response“) darauf.

- Im Allgemeinen steht jedes Datenelement in einer separaten Zeile (bestimmte eng zusammenhängende Elemente wie Tumorlokalisation und Art der Resektion können in einer Zeile zusammengefasst werden).

- Der Bericht muss alle obligatorischen Datenelemente (gemäß dem jeweiligen Protokoll) enthalten.
Hingegen werden keine Vorgaben gemacht zur Reihenfolge der Datenelemente, zu etwaigen zusätzlichen Elementen, zu ergänzenden Abschnitten in narrativem Format (d.h. in fortlaufendem Text) oder zu weiteren Aspekten der Formatierung.

\section{Standardisiert, strukturiert, synoptisch?}

Die Verwendung der Begriffe "standardisiert", "strukturiert" und "synoptisch" ist in der Literatur leider nicht ganz einheitlich. Das College of American Pathologists, welches die längste Tradition auf diesem Gebiet hat, verwendet systematisch den Begriff "synoptisch" und bezieht in dessen Definition auch Aspekte der Formatierung ein. Nach einer Klassifikation von Ellis und Srigley [4] definiert jedoch ein "synoptisches" Format ein mittleres Niveau der Strukturierung (-Abb. 1). Für Berichte, die zusätzlich eine zugrunde liegende Datenbankstruktur haben, verwenden sie den Begriff „standardisiertes strukturiertes Berichten".

Angesichts seiner Verbreitung in der englischsprachigen Literatur verwenden die Autoren in diesem Artikel vorzugsweise den Begriff "synoptisch“, zumal das 
Tab. 1 Übersicht der obligaten ("core") und fakultativen (,non-core") Parameter des ICCR-Protokolls für kolorektale Karzinome (Resektion)

Elemente des synoptischen Formats für das kolorektale Karzinom (ICCR-Dataset) Obligate (",core") Elemente

Neoadjuvante Therapie

Operatives Verfahren

Tumorlokalisation

Maximale Tumorgröße

Makroskopische Tumorperforation

Bezug zur anterioren peritonealen

Umschlagfalte $^{\mathrm{a}}$

Ebene der mesorektalen Exzision $^{\mathrm{a}}$

Histologischer Tumortyp

Histologischer Differenzierungsgrad

Tumorausdehnung

Lymphatische/venöse Gefäßinfiltration

Perineurale Infiltration

Lymphknotenstatus

Tumor deposits

Ansprechen auf neoadjuvante Therapie

Resektionsstatus

Histologisch bestätigte Fernmetastasen

TNM-Klassifikation

Fakultative („non-Core") Elemente

Klinische Informationen

Ebene der Sphinkterexzision ${ }^{\mathrm{a}}$

Ebene der mesokolischen Exzision

Tumorausbreitung über die Muscularis

propria hinaus

Tumor Budding

Sonstige pathologische Befunde

Ergänzende immunhistochemische Informationen (nur Coreelement für neuroendokrine Neoplasien)

ICCR International Collaboration for Cancer

Reporting

anur relevant beim rektalen Karzinom

synoptische Format das offensichtlichste Merkmal dieser Art von Berichten ist.

\section{Vollständigere Berichte durch synoptisches Format}

Neben einer Vereinheitlichung der Terminologie ist eine verbesserte Vollständigkeit bezogen auf prognostisch oder therapeutisch wichtige Parameter das wichtigste Ziel synoptischer Berichte. Dass dieses Ziel durch die Einführung synoptischer Berichte tatsächlich erreicht wird, ist in der Literatur gut belegt [6]. Eine Vielzahl von Stu- dien zeigt diesen Effekt beispielsweise für maligne Melanome [5-7], kolorektale Karzinome [10] oder Prostatakarzinome [1]. Eine Metaanalyse fand eine verbesserte Vollständigkeit von Berichten in 32 von 33 analysierten Studien [12]. Interessanterweise scheint ein synoptisches Format die Vollständigkeit von Berichten unabhängig davon zu verbessern, ob es sich um im entsprechenden Teilgebiet spezialisierte Pathologen handelt oder nicht [7, 10].

Für praktische Zwecke kann ferner auch schlicht der Aspekt relevant sein, dass mittels synoptischer Berichte alle relevanten Parameter an einem Ort zusammengefasst sind. Dies ist vor allem von Bedeutung, wenn die entsprechenden Proben (z.B. das resezierte Organ und die lokoregionären Lymphknoten) mit verschiedenen Antragsformularen zu verschiedenen Zeitpunkten eingesandt werden. Bei solchen parallelen Einsendungen können die synoptischen Protokolle erfahrungsgemäß sehr hilfreich zu sein, um beim Verfassen der Berichte oder der Präsentation im Tumorboard nicht die Übersicht zu verlieren.

\section{Quellen für synoptische Tumorprotokolle}

Aktuell existieren 2 größere Organisationen, die Protokolle für synoptische Berichte publizieren. Das CAP stellt nahezu 100 verschiedene Protokolle zur Verfügung, darunter neben Protokollen für Tumorresektionen auch solche für Biopsien bzw. Biomarker. Die Protokolle werden regelmäßig aktualisiert und sind kostenfrei auf der Internetseite der CAP [3] verfügbar. Ihre Verwendung ohne Lizenzierung unterliegt jedoch bestimmten Einschränkungen im Hinblick auf die Tiefe der Softwareintegration. Die Verwendung der Protokolle für Resektate ist für CAP-akkreditierte Institute obligatorisch. Es existieren verschiedene kommerzielle US-amerikanische Anbieter von Softwareschnittstellen zu Pathologielabor-Informationssystemen, nach Kenntnis der Autoren jedoch weder in deutscher Sprache noch kompatibel mit hierzulande verbreiteten Pathologiesystemen.

Die jüngere der beiden großen Herausgeberinnen von synoptischen Proto- kollen ist die International Collaboration on Cancer Reporting (ICCR) [13], eine gemeinsame Initiative verschiedener nationaler und internationaler Pathologieverbände, darunter auch die Deutsche Gesellschaft für Pathologie und die ÖsterreichischeGesellschaft für Pathologie. Bislang sind rund 50ICCR-Protokolleveröffentlicht, weitere sind in Vorbereitung. Grundsätzlich werden die Protokolle auf Englisch verfasst, für einzelne Protokolle sind aktuell autorisierte französische, spanische bzw. portugiesische Übersetzungen verfügbar. Die Protokolle werden als PDF-Dokumente publiziert, zusätzlich sind WordDokumente mit dem Inhalt der Protokolle verfügbar. ICCR gestattet eine weitgehend freie Nutzung der Protokolle für diagnostische Berichte und nicht kommerzielle Forschung. Sowohl die Datensätze der ICCR als auch der CAP integrieren die Informationen aus den wichtigen tumorbezogenen Standardquellen wie den jeweils aktuellen WHO-Klassifikationen und den UICC/ AJCC-TNM-Klassifikationen. Daneben werden evidenzbasierte Ergebnisse von wissenschaftlichen Publikationen eingearbeitet, die auch in den umfassenden Referenzlisten enthalten sind. Die ICCR bezieht sich zusätzlich auch auf Guidelines nationaler Fachgesellschaften, wie z. B. des Royal College of Pathologists, des Royal College of Pathologists of Australasia und auch der CAP.

CAP und ICCR entwickeln die Protokolle jeweils in einem mehrstufigen Prozess, beruhend auf aktueller Evidenz, der Arbeit von Expertengremien und einer öffentlichen Konsultationsphase. Je nach Evidenz wird zwischen obligaten und fakultativen Datenelementen unterschieden, wobei im Detail durchaus relevante Unterschiede zwischen den jeweiligen Protokollen der beiden Herausgeberinnen bestehen können. Wie bereits erwähnt, wird eine Abstimmung mit den jeweiligen Auflagen der WHO-Klassifikation und der TNM-Klassifikation angestrebt.

\section{Informationstechnische Umsetzung}

Im Idealfall sind die Vorlagen und Datensätze in einem elektronischen Befundsystem hinterlegt und können auch elektronisch ausgefüllt werden. In der Praxis 
Sigmaresektat mit einem mäßiggradig differenzierten Adenokarzinom (maximaler Durchmesser 4,6 cm) mit Infiltration sämtlicher Wandschichten bis ins perikolische Fettgewebe, mit max. $7 \mathrm{~mm}$ tiefer Invasion jenseits der Muscularis propria, ohne Nachweis einer Perforation. Nachweis von bis zu 14 Tumorbuds in 20-facher Vergrößerung an der Tumorperipherie. Nachweis einer intra-und extramuralen lymphatischen und venösen Gefäßinfiltration. Kein Nachweis einer Perineuralscheideninfiltration.

Tumorfreier oraler und aboraler Resektionsrand. Tumorfreier Gefäßabsetzungsrand. Die Tumorresektion in der Mesocolischen Ebene erfolgt.

Nachweis von 3 Lymphknotenmetastasen in 25 untersuchten regionären Lymphknoten. Kein Nachweis von Tumordeposits.

Daneben 5 tubuläre Adenome mit niedriggradiger Dysplasie

In zusätzlich durchgeführten immunhistochemischen Untersuchungen zeigte sich eine erhaltene Expression der DNA-Reparatur-Proteine MLH1, MSH2, PMS2, und MSH6 TNM-Klassifikation (UICC, 8. Auflage, 2017): pT3 pN1b (3/25) L1 V1 PnO G2 R0.

a

Klinische Information: Sigmakarzinom, bioptisch gesichert

Neoadjuvante Therapie: keine durchgeführt

Operatives Verfahren: Sigmaresektion

Tumorlokalisation: Sigma

Maximale Tumorgröße: $4,6 \mathrm{~cm}$

Makroskopische Tumorperforation: nicht nachgewiesen

Level der Mesocolischen Exzision: mesocolisch

Histologischer Tumortyp: Adenokarzinom

Histologischer Differenzierungsgrad: mäßiggradig differenziert (G2)

Tumorausdehnung: bis ins perikolische Fettgewebe

Infiltration über die Muscularis propria hinaus: $7 \mathrm{~mm}$

Tumorbudding: Bd3 (14 buds)

Lymphatische/venöse Gefäßinfiltration: vorhanden (intra- und extramural)

Perineurale Infiltration: nicht nachgewiesen

Resektionsränder

Oraler Resektionsrand: tumorfrei

Aboraler Resektionsrand: tumorfre

Gefässabsetzungsrand: tumorfrei

Lymphknotenstatus

Anzahl untersuchter Lymphknoten: 25

Anzahl befallener Lymphknoten: 3

Tumor deposits: nicht nachgewiesen

Zusätzliche Untersuchungen: Erhaltene Expression der DNA-Reparatur-Proteine

(Immunhistochemie, MLH1, MSH2, PMS2, MSH6)

sonstige pathologische Befunde: 5 tubuläre Adenome mit niedriggradiger Dysplasie

Histologisch bestätigte Fernmetastasen: nicht nachgewiesen

TNM-Klassifikation (UICC, 8. Auflage, 2017): pT3 pN1b (3/25) L1 V1 PnO G2 R0

b

Abb. $2 \triangle$ Narrative (a) und synoptische (b) Darstellung eines Pathologieberichts. Korrespondierende Datenelemente sind durch die jeweils gleiche Farbe dargestellt. Trotz der einfachen Formatierung sind die Datenelemente im synoptischen Format leichter auffindbar. Im einfachsten Fall könnte ein solcher Beispielbericht als Diktatvorlage verwendet werden, was die Umsetzung synoptischer Berichte (Stufe 3 nach Ellis und Srigley [4]) ohne spezifische IT-Implementierung ermöglichen würde

werden jedoch Pathologiebefunde häufig noch immer als Textdokument oder Text innerhalb eines in Blöcken aufgebauten Befundsystems verfasst. Die Implementierung der vorgeschlagenen Datensätze in den Pathologiebefund kann inhaltlich und formell bereits auf einer solchen Textebene erfolgen und wäre somit in Level 3 entsprechend der in - Abb. 1 dargestellten Kategorisierung anzusiedeln. Für eine inhaltlich standardisierte Befundung können institutionalisierte Berichtsvorlagen verwendet oder es kann mit Textbausteinen gearbei- tet werden (Beispiel - ICCR-Datensatz Einarbeitung in Fließtext - synoptischer Bericht). Derartige Vorlagen können dann auch bereits die Grundlagen für IT-basierte Lösungen sein.

Nicht zu unterschätzen ist allerdings der Aufwand, nicht nur für eine erstmalige Implementierung eines breiten Spektrums an synoptischen Berichtsvorlagen, sondern vor allem für deren kontinuierliche Aktualisierung. Hier dürften auch die Ressourcen auch größerer Pathologischer Institute rasch überfordern sein. Außer- dem wäre der betreffende Aufwand jeweils weitgehend durch die jeweilige Pathologie zu leisten, während andere Fachdisziplinen (und auch Forschung sowie Krankenhausverwaltung) mindestens im gleichen Umfang profitieren würden.

Mittel- bis längerfristig scheint daher eine gewissen Professionalisierung der betreffenden IT-Lösungen unumgänglich, ähnlich wie aktuell bereits in den USA oder auch den Niederlanden. Dabei sind die Modelle durchaus unterschiedlich. In den USA existiert eine Reihe von kommerziellen Anbietern die lizenzierte, auf den CAP-Protokollen basierende Softwarelösungen anbieten, die wiederum über Schnittstellen zu den gängigen Pathologie-Informationssystemen verfügen. Dagegen sind in den Niederlanden sowohl die Entwicklung als auch die informationstechnische Umsetzung synoptischer Berichte in der Hand eines nicht gewinnorientierten Anbieters, der Stiftung „Pathologisch-Anatomisch Landelijk Geautomatiseerd Archief" (PALGA) [11].

Eine gewisse zusätzliche Herausforderung für eine Implementierung synoptischer Berichte im deutschsprachigen Raum ergibt sich daraus, dass bislang keine autorisierten deutschen Übersetzungen der gängigen Protokolle existieren [2]. Während die Protokolle der CAP ausschließlich auf Englisch angeboten werden, hat ICCR begonnen, offizielle Übersetzungen zu publizieren (bislang auf Spanisch, Portugiesisch und Französisch).

\section{ICCR-Protokolle für gastro- intestinale Tumoren}

In jüngerer Vergangenheit hat ICCR eine Reihe von Protokollen zu gastrointestinalen Tumoren publiziert. Zum Zeitpunkt der Verfassung dieses Manuskripts waren dies Protokolle für Resektionen von Karzinomen des exokrinen Pankreas, des Ösophagus, des Magens, des Kolons und Rektums sowie für intrahepatische/perihiläre Cholangiokarzinome bzw. hepatozelluläre Karzinome. Weiterhin existieren spezifische Protokolle für endoskopische Resektionen von Karzinomen des Ösophagus und gastroösophagealen Übergangs, des Magens und für Polypektomien aus Kolon und Rektum vor. Die Protokolle sind über die Homepage der ICCR öffentlich zugänglich und 
können ohne Lizenzgebühren verwendet werden. Die sukzessive Veröffentlichung der Protokolle auf der Homepage der ICCR wird von Publikationen in internationalen Fachzeitschriften begleitet $[8,9]$.

Ein wesentlicher Unterschied zu den CAP-Protokollen besteht darin, dass die Protokolle der ICCR noch systematischer und detaillierter klinische Daten dokumentieren, die eine prognostische oder differenzialdiagnostische Bedeutung besitzen. Hieraus könnte sich die interessante Perspektive ergeben, die betreffenden Daten bereits bei der Einsendung der Proben in standardisierter Form zu erheben. Vor allem wenn eine elegante Einbindung in Klinikinformationssysteme vorhanden wäre, würde dies nicht nur uns Pathologen unsere Arbeit erleichtern, sondern einen echten Mehrwert für die Qualität der Patientenversorgung bedeuten.

Die ICCR-Protokolle unterscheiden obligate Parameter („,core items ${ }^{\prime \prime}$ ) von fakultativen („,non-core items"). In • Tab. 1 wird dies am Beispiel des Protokolls für kolorektale Karzinome dargestellt; - Abb. 2 illustriert anhand des Beispiels eines Sigmakarzinoms eine mögliche konkrete Umsetzung des betreffenden ICCR-Protokolls im Vergleich zu einem inhaltlich übereinstimmenden narrativen Bericht.

\section{Ausblick}

Bereits die ersten Schritte in Richtung stärker strukturierter und standardisierter Berichte (bis Stufe 3 nach Ellis und Srigley [4]) bieten einen Nutzen für den jeweiligen Patienten und das für seine Behandlung verantwortliche interdisziplinäre Team. Besonders deutlich wird dies im Rahmen des interdisziplinären Tumorboards, wo die Vollständigkeit der Berichte und deren eindeutige Terminologie entscheidend zu einer optimalen Entscheidungsfindung beitragen können. Nebenbei bemerkt ist es umso erfreulicher, dass das Konzept der synoptischen Berichte von der Pathologie auch auf andere Disziplinen überzugreifen beginnt [6].

Ein erheblich über den einzelnen Patienten hinausreichendes Potenzial ergibt sich hingegen vor allem durch die Stufen 4-6 nach Ellis and Srigley [4], da sich hieraus wesentliche Impulse für die Qualitätssicherung, sowie über eine Integration

\section{Standardized structured reports for gastrointestinal tumors}

Synoptic reporting in pathology as opposed to traditional narrative reporting is defined by a laboratory value-like format and use of standardized checklists. It contributes to completeness and comprehensibility of pathology reports and ultimately patient care. As of today, two major institutions publish synoptic reporting templates, the College of American Pathologists (CAP) and the International Collaboration for Cancer Reporting (ICCR). Synoptic protocols are available for all major cancer types and provide not only a standardized terminology and a checklist for completeness of reports, but also facilitate uniform utilization of diagnostic criteria. Additionally, both CAP and ICCR protocols are accompanied by detailed and up-to-date reference lists and thereby represent a valuable source of information even when synoptic reporting is not used. The benefits and challenges of implementation of synoptic reporting are discussed, in particular with regard to reporting in German.

\section{Keywords}

Checklist · Data accuracy $\cdot$ Healthcare quality assurance $\cdot$ Pathology $\cdot$ Quality control

mit epidemiologischen Krebsregistern und Biobanken auch für verschiedenste wissenschaftliche Fragestellungen ergeben.

\section{Fazit für die Praxis}

- Synoptische Berichte tragen zur Standardisierung und verbesserten Vollständigkeit von Pathologiebefunden bei.

- International Collaboration for Cancer Reporting (ICCR) und College of American Pathologists (CAP) stellen eine Vielzahl aktueller und evidenzbasierter Protokolle zur Verfügung.

- Die praktische Umsetzung im deutschsprachigen Raum ist zurzeit noch anspruchsvoll, da autorisierte deutsche Übersetzungen bislang fehlen.

- Auch wer synoptische Berichte (noch) nicht verwendet, findet in den Protokollen eine Fülle an aktuellen und relevanten Informationen zu den betreffenden Tumortypen.

\section{Korrespondenzadresse}

\section{Prof. Dr. Ekkehard Hewer}

Institut universitaire de pathologie, Centre hospitalier universitaire vaudois (CHUV) et Université de Lausanne

Rue du Bugnon 25, 1011 Lausanne, Schweiz ekkehard.hewer@unil.ch

Funding. Open access funding provided by University of Lausanne

\section{Einhaltung ethischer Richtlinien}

Interessenkonflikt. E. Hewer, A. Rump und R. Langer geben an, dass kein Interessenkonflikt besteht.
Für diesen Beitrag wurden von den Autoren keine Studien an Menschen oder Tieren durchgeführt. Für die aufgeführten Studien gelten die jeweils dort angegebenen ethischen Richtlinien.

Open Access. Dieser Artikel wird unter der Creative Commons Namensnennung 4.0 International Lizenz veröffentlicht, welche die Nutzung, Vervielfältigung, Bearbeitung, Verbreitung und Wiedergabe in jeglichem Medium und Format erlaubt, sofern Sie den/die ursprünglichen Autor(en) und die Quelle ordnungsgemäß nennen, einen Link zur Creative Commons Lizenz beifügen und angeben, ob Änderungen vorgenommen wurden.

Die in diesem Artikel enthaltenen Bilder und sonstiges Drittmaterial unterliegen ebenfalls der genannten Creative Commons Lizenz, sofern sich aus der Abbildungslegende nichts anderes ergibt. Sofern das betreffende Material nicht unter der genannten Creative Commons Lizenz steht und die betreffende Handlung nicht nach gesetzlichen Vorschriften erlaubt ist, ist für die oben aufgeführten Weiterverwendungen des Materials die Einwilligung des jeweiligen Rechteinhabers einzuholen.

Weitere Details zur Lizenz entnehmen Sie bitte der Lizenzinformation auf http://creativecommons.org/ licenses/by/4.0/deed.de.

\section{Literatur}

1. Aumann K, Amann D, Gumpp V, Hauschke D (2012) Template-based synoptic reports improve the quality of pathology reports of prostatectomy specimens

2. Banz Y, Berezowska S, de Leval Let al (2018) Advancing synoptic cancer reports beyond English: the University of Bern/PathoLink approach. Virchows Arch 473:655-656

3. College of American Pathologists (2017) Cancer protocol templates. https://www.cap.org/ protocols-and-guidelines/cancer-reportingtools/cancer-protocol-templates. Zugegriffen: 24. Juni 2021

4. Ellis DW, Srigley J (2016) Does standardised structured reporting contribute to quality in diagnostic pathology? The importance of evidence-based datasets. Virchows Arch 468:51-59 
5. Haydu LE, Holt PE, Karim RZ et al (2010) Quality of histopathological reporting on melanoma and influence of use of a synoptic template. Histopathology 56:768-774

6. Hewer E (2020) The oncologist's guide to synoptic reporting: a primer. Oncology 98:396-402

7. Karim RZ, van den Berg KS, Colman MH et al (2008) The advantage of using a synoptic pathology report format for cutaneous melanoma. Histopathology. https://doi.org/10.1111/j.13652559.2007.02921.x

8. Lam AK, Bourke MJ, Chen R et al (2021) Dataset for the reporting of carcinoma of the esophagus in resection specimens: recommendations from the International Collaboration on Cancer Reporting. Hum Pathol 114:54-65

9. Loughrey MB, Webster F, Arends MJ et al (2021) Dataset for pathology reporting of colorectal cancer: recommendations from the International Collaboration on Cancer Reporting (ICCR). Ann Surg. https://doi.org/10.1097/SLA. 0000000000005051

10. Messenger DE, McLeod RS, Kirsch R (2011) What impact has the introduction of a synoptic report for rectal cancer had on reporting outcomes for specialist gastrointestinal and nongastrointestinal pathologists? Arch Pathol Lab Med 135:1471-1475

11. Palga (2021) Palga Home. https://www.palga.nl/. Zugegriffen: 15. Aug. 2021
12. Sluijter CE, van Lonkhuijzen LRCW, van Slooten H-J et al (2016) The effects of implementing synoptic pathology reporting in cancer diagnosis: a systematic review. Virchows Arch 468:639-649

13. International Collaboration on Cancer Reporting (2021) Homepage. http://www.iccr-cancer.org/. Zugegriffen: 16.Aug. 2021

\section{Online auf alle CME-Fortbildungskurse von SpringerMedizin.de zugreifen}

Gestalten Sie Ihre Fortbildung nach Ihren Bedürfnissen: e.Med Interdisziplinär - das Kombi-Abo von SpringerMedizin.de - bietet Ihnen Online-Zugang zu über 500 CME-Fortbildungskursen aller Fachrichtungen. Sie alleine entscheiden, welche Kurse Sie für Ihre Fortbildung nutzen möchten.

e.Med Interdisziplinär bietet Ihnen viele Vorteile:

- Stellen Sie Ihre individuelle Fortbildung einfach und komfortabel selbst zusammen.

- Sie können Ihren Lernerfolg jederzeit messen und in Form von CME-Punkten und Teilnahmebescheinigungen dokumentieren.

- Jeden Monat kommen rund 50 neue CME-Kurse aus den Fachzeitschriften von Springer Medizin hinzu.

- Unser Service für Sie: Nach erfolgreicher Teilnahme werden die CME-Punkte automatisch an die Bundesärztekammer übermittelt.

- Auf Wunsch erhalten Sie mit e.Med Interdisziplinär darüber hinaus eine gedruckte Fachzeitschrift Ihrer Wahl.
Mit den fachspezifischen e.Med-Abos können

Sie sich effizient innerhalb Ihres Fachgebietes auf dem Laufenden halten. Das Angebot reicht von AINS bis Radiologie - Sie haben die Wahl!

Testen Sie e.Med kostenlos und unverbindlich!

Jetzt informieren unter

www.springermedizin.de $\rightarrow$ „Abo-Shop"

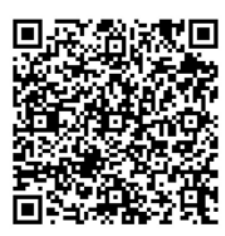

oder telefonisch unter 0800-77 80777

(Montag bis Freitag, 10 bis $17 \mathrm{Uhr}$ ) 